\title{
Intersection of Generic Rotations in Some Classical Spaces
} by

\author{
Krzysztof NOWAK and Grzegorz TOMKOWICZ \\ Presented by Stanistaw KWAPIEN
}

\begin{abstract}
Summary. Consider an o-minimal structure on the real field $\mathbb{R}$ and two definable subsets $A, B$ of the Euclidean space $\mathbb{R}^{n}$, of the unit sphere $\mathbb{S}^{n}$ or of the hyperbolic space $\mathbb{H}^{n}, n \geq 2$, which are of dimensions $k, l \leq n-1$, respectively. We prove that the dimension of the intersection $\sigma(A) \cap B$ is less than $\min \{k, l\}$ for a generic rotation $\sigma$ of the ambient space; here we set $\operatorname{dim} \emptyset=-1$.
\end{abstract}

1. Introduction. This paper is devoted to generic intersections of rotations in the three classical spaces: the Euclidean space $\mathbb{R}^{n}$, the unit sphere $\mathbb{S}^{n}$ and the hyperbolic space $\mathbb{H}^{n}$ with $n \geq 2$. From now on, $\mathbb{X}^{n}$ will stand for one of those spaces.

By a rotation of $\mathbb{X}^{n}$ we mean an orientation preserving isometry of $\mathbb{X}^{n}$ whose set of fixed points is an $(n-2)$-dimensional subspace $H$ of $\mathbb{X}^{n}$. Given such a subspace $H$ and an angle, there are at most two rotations of $\mathbb{X}^{n}$ around $H$ by that angle. Let $\mathfrak{R}_{n}$ denote the set of all rotations of $\mathbb{X}^{n}$ with its natural topology. It may be regarded as an algebraic submanifold of the linear group of all isometries of $\mathbb{X}^{n}$, which, as is easily seen, has dimension $2 n-1$.

Consider an o-minimal expansion $\Sigma$ of the field $\mathbb{R}$ of real numbers. By definable sets we shall always mean sets definable with parameters with respect to the structure $\Sigma$. This paper is related to the articles [3, 4, and its main result, stated below, partially generalizes the theorem on generic intersections from the former article, because the set of rotations does not have a group structure (under composition).

2010 Mathematics Subject Classification: Primary 03C64; Secondary 51M05, 51M10.

Key words and phrases: Euclidean space, hyperbolic space, rotations, o-minimal structure, intersections of definable sets.

Received 30 September 2016.

Published online 21 October 2016. 
TheOrem 1.1. If $A, B \subset \mathbb{X}^{n}$ are non-empty definable subsets of dimensions $k, l<n$, respectively, then there exists a definable nowhere dense subset $Z$ of $\mathfrak{R}_{n}$ such that

$$
\operatorname{dim}(\sigma(A) \cap B)<\min \{k, l\} \quad \text { for all } \sigma \in \mathfrak{R}_{n} \backslash Z .
$$

This theorem is inspired by and applied in [2], devoted to a concept of a small set, a refinement of the notion of a Tarski nullset.

2. Proof of Theorem 1.1. The proof given here is an adaptation of the proof of the theorem on generic intersections in [3. We need an elementary proposition relying on definable cell decomposition, which can be found e.g. in [1, Chap. 4, Proposition 1.5].

Proposition 2.1. Let $f: V \rightarrow W$ be a definable map between non-empty definable sets. Then

$$
\begin{aligned}
& \operatorname{dim} f^{-1} f(v) \leq k \text { for all } v \in V \Rightarrow \operatorname{dim} V \leq k+\operatorname{dim} f(V), \\
& \operatorname{dim} f^{-1} f(v) \geq k \text { for all } v \in V \Rightarrow \operatorname{dim} V \geq k+\operatorname{dim} f(V) .
\end{aligned}
$$

Now let

$$
X:=\mathbb{X}^{n} \times \mathbb{X}^{n}, \quad \mathcal{X}:=\left\{(\sigma, x, y) \in \mathfrak{R}_{n} \times X: y=\sigma(x)\right\},
$$

and let

$$
p: \mathcal{X} \rightarrow \mathfrak{R}_{n} \text { and } q: \mathcal{X} \rightarrow X
$$

be the canonical projections. Clearly, the set $\mathcal{X}$ and the maps $p$ and $q$ are definable. It is not difficult to calculate the dimension of the fibres $q^{-1}(x, y)$ :

$$
\begin{array}{ll}
\operatorname{dim} q^{-1}(x, y)=n-1 & \text { if } x \neq y, \\
\operatorname{dim} q^{-1}(x, y)=2 n-3 & \text { if } x=y .
\end{array}
$$

Without loss of generality we may assume that $k \leq l$. We can partition $A \times B$ into two sets:

$$
A \times B=(A \times B \backslash \Delta) \cup(A \times B \cap \Delta),
$$

where $\Delta \subset X$ is the diagonal of $X$.

Proposition 2.1 along with formulae 2.1) and 2.2 yields

$\operatorname{dim} q^{-1}(A \times B \backslash \Delta) \leq k+l+n-1 \leq k+2 n-2=k-1+\operatorname{dim} \Re_{n}$,

$\operatorname{dim} q^{-1}(A \times B \cap \Delta) \leq k+2 n-3<k-1+\operatorname{dim} \mathfrak{R}_{n}$.

Hence

$$
\operatorname{dim} \mathcal{E} \leq k-1+\operatorname{dim} \mathfrak{R}_{n} \quad \text { where } \quad \mathcal{E}:=q^{-1}(A \times B) \subset \mathcal{X} .
$$

Again it follows from Proposition 2.1 that the definable subset

$$
Z:=\left\{\sigma \in \mathfrak{R}_{n}: \operatorname{dim}\left(p^{-1}(\sigma) \cap \mathcal{E}\right)>k-1\right\}
$$

is of dimension $<\operatorname{dim} \mathfrak{R}_{n}$, and thus is a nowhere dense subset of $\mathfrak{R}_{n}$. 
Further, let $\pi: \Delta \rightarrow \mathbb{X}^{n}$ be the projection onto the first factor. Then

$$
\begin{aligned}
\sigma(A) \cap B & =\pi((\sigma(A) \times B) \cap \Delta) \\
& =\pi \circ\left(\sigma \times \operatorname{Id}_{\mathbb{X}^{n}}\right)\left((A \times B) \cap\left\{(x, \sigma(x)): x \in \mathbb{X}^{n}\right\}\right),
\end{aligned}
$$

whence the sets $\sigma(A) \cap B$ and

$$
\{\sigma\} \times\left[(A \times B) \cap\left\{(x, \sigma(x)): x \in \mathbb{X}^{n}\right\}\right]=p^{-1}(\sigma) \cap \mathcal{E}
$$

are definably homeomorphic. Therefore

$$
\operatorname{dim}(\sigma(A) \cap B) \leq k-1 \quad \text { for all } \sigma \in \mathfrak{R}_{n} \backslash Z
$$

which completes the proof.

\section{References}

[1] L. van den Dries, Tame Topology and O-minimal Structures, Cambridge Univ. Press, 1998.

[2] J. Mycielski and G. Tomkowicz, On small subsets in Euclidean spaces, Bull. Polish Acad. Sci. Math. 64 (2016), 109-118.

[3] K. J. Nowak, A theorem on generic intersections in an o-minimal structure, Fund. Math. 227 (2014), 21-25.

[4] J. Mycielski and K. Nowak, On intersections of generic perturbations of definable sets, Bull. Polish Acad. Sci. Math. 64 (2016), 95-103.

Krzysztof Nowak

Institute of Mathematics

Faculty of Mathematics and Computer Science

Jagiellonian University

Łojasiewicza 6

30-348 Kraków, Poland

E-mail: nowak@im.uj.edu.pl
Grzegorz Tomkowicz Centrum Edukacji $G^{2}$ Moniuszki 9 41-902 Bytom, Poland E-mail: gtomko@vp.pl 
